Revue d'histoire de l'Amérique française

FG REVUE D.HISTOIRE DE L'AMÉRIQUE FRANÇAISE

\title{
Impulsion donnée par Mgr Bourget à la pratique religieuse
}

\section{Léon Pouliot}

Volume 16, numéro 1, juin 1962

URI : https://id.erudit.org/iderudit/302171ar

DOI : https://doi.org/10.7202/302171ar

Aller au sommaire du numéro

Éditeur(s)

Institut d'histoire de l'Amérique française

ISSN

0035-2357 (imprimé)

1492-1383 (numérique)

Découvrir la revue

Citer cet article

Pouliot, L. (1962). Impulsion donnée par Mgr Bourget à la pratique religieuse. Revue d'histoire de l'Amérique française, 16(1), 66-80.

https://doi.org/10.7202/302171ar d'utilisation que vous pouvez consulter en ligne.

https://apropos.erudit.org/fr/usagers/politique-dutilisation/ 


\section{IMPULSION DONNÉE PAR MGR BOURGET À LA PRATIQUE RELIGIEUSE *}

On m'a demandé de parler de l'impulsion donnée par Mgr Bourget à la pratique religieuse. L'expression me paraît particulièrement bien choisie. Elle indique qu'il y a continuité, que le mérite propre de Mgr Bourget, n'est pas d'avoir créé de toutes pièces, mais d'avoir ranimé, organisé, intensifié une foi et une pratique religieuse qui étaient déjà le fait de la vaste majorité dans le diocèse de Montréal.

\section{I \\ LA SITUATION EN 1840}

Il y avait cependant des ombres au tableau; et en voici les principales causes. De 1821 à 1836 le clergé et les fidèles avaient été âprement divisés autour de la condition faite à $\mathrm{Mgr}$ Lartigue à Montréal. Sous le titre d'évêque de Telmesse in partibus et de vicaire général, il était en droit le dédoublement parfait à Montréal de l'évêque de Québec. Mais il ne l'était pas en fait. Les Sulpiciens, seigneurs temporels et spirituels de Montréal, ne reconnaissaient pas son autorité; ils l'ignoraient et s'adressaient directement à l'évêque de Québec pour la solution de leurs problèmes. Ils subissaient de mauvaise grâce sa présence dans la ville et le lui faisaient sentir; ils entraînaient à leur suite les communautés religieuses dont ils étaient les aumôniers et une partie des fidèles. Cette hostilité ouverte et prolongée entre les chefs spirituels de la ville n'était pas sans poser des problèmes à la conscience des fidèles; et elle entre pour quelque chose dans la diminution de la pratique religieuse et du nombre des pratiquants.

Pendant la même période, 1821-1836, la population croît rapidement tant à la ville qu'à la campagne. Pour répondre à

* Travail présenté lors de la réunion générale de l'Institut d'Histoire de l'Amérique française, le 14 avril 1962. 
tant de besoins, il faudrait un clergé plus nombreux. Mgr Lartigue expose la situation à l'évêque de Québec. Il demande et redemande des prêtres; mais il n'est pas exaucé selon la mesure de ses désirs. Il a l'impression très nette que, eu égard aux besoins de chaque région, il y a trop de prêtres à Québec, pas assez à Montréal. Il semble qu'il ait raison. Et c'est une autre circonstance qui est préjudiciable à la pratique religieuse régulière et fervente.

En 1836, Mgr Lartigue devient évêque de Montréal. Mais son clergé est toujours trop peu nombreux. De plus, la propagande protestante qui s'exerce désormais en français, l'agitation politique dont Montréal est le centre, la diffusion des Paroles d'un croyant de Lamennais, l'attitude de Mgr Lartigue en face des Patriotes, sa condamnation du recours aux armes, la privation de la sépulture ecclésiastique aux Patriotes morts les armes à la main, autant d'événements qui expliquent une désaffection de la pratique religieuse, et même chez plusieurs, une désaffection de l'Église, dans le diocèse de Montréal. Deux textes de Mgr Bourget nous aideront à serrer la situation de plus près. Dans son Mandement d'entrée, qui porte la date du 3 mai 1840, il écrit:

Nous ne pouvons nous dissimuler qu'il y a des plaies profondes à guérir, des abus invétérés à corriger, des scandales déplorables à réprimer. Nous ne pouvons nous cacher que l'indifférence et l'irréligion, l'ivrognerie et les excès de la débauche ont fait parmi vous d'étranges ravages. Nous voyons avec une douleur inexprimable que plusieurs parmi vous vivent dans une coupable négligence des devoirs les plus sacrés de la religion; que beaucoup ont cessé de fréquenter les tribunaux de la pénitence et ne paraissent jamais à la table du Seigneur. Nous n'ignorons point que les jours du Seigneur sont indignement profanés par des jeux et des dissolutions de toute espèce, par des ventes et trafics sordides, à la honte des lois de l'Eglise et de l'Etat. Nous apprenons avec frayeur les efforts incroyables que font les ennemis de la foi pour pervertir les brebis dont le Seigneur nous a confié la garde, en répandant parmi vous des livres corrompus et en établissant 
des écoles qui, si elles étaient encouragées par vous, produiraient bientôt des fruits bien amers. Voilà ce qui multiplie nos craintes et nos alarmes.

Dira-t-on de ce texte qu'il appartient à un genre littéraire, et que dès lors, il est manifestement exagéré ? Tel qu'il est, il nous met en garde contre des généralisations rapides. Mgr Bourget ne parle pas d'un abandon général ou presque général de la pratique religieuse; il note que plusieurs ne s'acquittent plus de leurs devoirs les plus sacrés, que beaucoup sont infidèles à la confession et à la communion annuelles. Ce plusieurs et ce beaucoup, il nous est permis de les préciser davantage, grâce au second document de Mgr Bourget. De la Relation du diocèse de Montréal qu'il présente à la Propagande, lors de son premier voyage à Rome, en 1841, nous ne retiendrons ici que les chiffres essentiels :

Population catholique du diocèse

Communiants

186,244

$\mathrm{Ne}$ se confessent pas

115,071

$\mathrm{Ne}$ communient pas

Concubinaires

7,177

13,726

Apostats

23

En chiffres ronds, c'est un communiant sur vingt qui ne se confesse pas annuellement, et un sur dix qui ne reçoit pas la communion pascale. - Pour regrettables qu'ils soient, le nombre des concubinaires, 44, et le nombre des apostats, 23 , ne nous paraissent pas tellement supérieurs à la moyenne des autres diocèses du monde entier. Quant aux dangers provenant de la propagande hérétique, l'évêque note qu'il y a 50 chapelles protestantes, 43 ministres, 41 écoles fréquentées à la campagne par 829 élèves, dont 75 enfants catholiques. Il n'a pas de statistiques pour la ville, mais il reconnaît que les élèves catholiques inscrits aux institutions protestantes sont plus nombreux qu'à la campagne, parce que les écoles catholiques de langue anglaise sont trop rares et mal organisées. Affaiblie au dedans par une pratique religieuse à la baisse, menacée du dehors par une propagande protestante qui dispose d'un personnel nombreux et de moyens financiers imposants, telle nous apparaît l'Église de Montréal en ces années 1840-1841. 


\section{II}

\section{LA RÉACTION CATHOLIQUE}

\section{LA PERSONNALITÉ DE MGR BouRget}

Nous nous contenterons d'indiquer ici les principaux éléments de la réaction catholique de Montréal. Il y a d'abord la personnalité de Mgr Bourget, infatigable au travail et toute de feu, parce qu'il a de sa charge pastorale une très haute et très exigeante conception. A titre d'exemple, voyons-le à l'œuvre pendant la première année de son gouvernement.

Le 3 mai 1840, mandement d'entrée; le 4, mandement d'institution des Filles de la Charité de Saint-Hyacinthe (Sœurs Grises), document d'une rare élévation de pensée. Le 5 juin, touchante lettre à $M$. Joseph Papineau, père du grand tribun populaire, qui a manifesté le désir de revenir à la pratique religieuse. «Je vous prie de croire qu'en écrivant la présente à un homme dont j'ai toujours admiré les talents, je ne consulte que les grands intérêts de son âme. Je vous conjure, au nom de Jésus-Christ, qui est venu pour nous sauver tous, de ne pas craindre de venir vous jeter dans les bras d'un homme plus pécheur que vous.» Il interrompra sa retraite annuelle pour recevoir M. Papineau, et il l'assure que, dès la première rencontre, toutes ses difficultés s'évanouiront.

$\mathrm{Du} 7$ juin au 20 juillet, l'évêque de Montréal visite 15 paroisses de la vallée du Richelieu; il prend sa large part des prédications et des confessions; il se rend compte par lui-même de la décence ou de l'indécence des lieux et objets de culte, de la propreté des cimetières; il laisse à chaque endroit un Mémorial, liste d'abus à corriger ou de bons usages à conserver. Il administre le sacrement de confirmation à 5,292 personnes. En septembre et octobre de la même année, il fait la visite des chantiers de l'Ottawa, où des centaines de jeunes gens du diocèse sont, à la grande tristesse de leurs parents, privés de tout secours religieux. Il bénit huit chapelles, érige huit nouvelles missions, donne la confirmation à neuf cents personnes et a le bonheur d'en voir 1750 s'approcher de la Sainte table. «De cette visite, écrit le $P$. Alexis, date l'organisation religieuse de la vallée de l'Ottawa. » 
Du 22 décembre 1840 au début de janvier 1841, c'est dans les lointaines missions ou paroisses de l'actuel diocèse de Valleyfield que Mgr Bourget dirige ses pas. En février, nous le retrouvons à Contrecœur et à Berthier où il préside les exercices de retraites populaires; et il se rend jusqu'à Rawdon pour la visite pastorale.

Quand on songe à l'inconfort des routes et des moyens de transport de l'époque pendant l'hiver, on comprend que le zèle de Mgr Bourget ait été taxé d'imprudence. Mgr Turgeon, coadjuteur de Québec, lui écrit: "Vos bons prêtres, ainsi que les évêques, vos frères, veulent vous conserver sur la terre, en dépit du désir que vous avez de vous en aller au ciel; voilà pourquoi ils n'aiment pas à vous voir faire des visites épiscopales en hiver, au risque d'y contracter de sérieuses maladies. » Ceux qui bénéficiaient du zèle de Mgr Bourget le trouvaient-ils intempestif ? Il ne semble pas. Et, au fond, tout le diocèse admirait cet évêque si entièrement donné à son ministère pastoral. Tout cela n'empêche pas Mgr Bourget de s'acquitter de ses autres devoirs; et ils sont nombreux et lourds en cette année 1840-1841. En mai 1840, il tient tête à l'autoritaire gouverneur-général Poulett Thomson. Prêtant l'oreille à des accusations fausses, celui-ci a exigé le déplacement de M. Joseph Marcoux, missionnaire des Iroquois de Caughnawaga. Avant d'obtempérer à la volonté du gouverneur, Mgr Bourget fait son enquête; et comme elle est favorable à M. Marcoux, il en informe Thomson. Celui-ci n'entend pas céder à l'évêque, et le combat s'engage entre l'Église et l'État. Soucis qui s'ajoutent à ceux de la visite pastorale des mois de juin et juillet. De longues lettres de $\mathrm{Mgr}$ Bourget s'envolent vers Montréal, Québec et Caughnawaga et l'on ne peut qu'admirer la ferme tranquillité de l'évêque et son esprit surnaturel. Il suffira de noter ici que M. Marcoux était encore et toujours missionnaire à Caughnawaga quand il décéda en 1855.

Fin août 1840, Mgr Bourget préside les exercices de la retraite sacerdotale. C'est là que fut décidée la fondation d'un journal qui aura pour but de compléter l'information religieuse des fidèles et de les protéger contre les dangers de la propagande protestante. En octobre, Mgr Bourget confie aux Sulpiciens la formation de son clergé: c'est la fondation du Grand Séminaire 
de Montréal. Par l'institution du Chapître, janvier 1841, il se donne des auxiliaires précieux dans l'administration du diocèse. Le 2 février, il établit comme dévotion propre au diocèse la dévotion au Très Saint et Immaculé Cœur de Marie, dont le siège est à Paris et qui a pour but la conversion des pécheurs. En un mot, Mgr Bourget donne au diocèse les structures, les organismes dont il avait besoin pour vivre et aller de l'avant, malgré des circonstances difficiles.

\section{INAUGURATION DES RETRAITES OU MISSIONS POPULAIRES PAR MGR DE FORBIN-JANSON}

Tous les efforts de Mgr Bourget n'auraient eu qu'un succès relatif, si un événement ne s'était produit alors, que l'évêque de Montréal désirait ardemment, mais dont il n'a pas la paternité. Nous voulons parler de la venue au Canada de Mgr de ForbinJanson, évêque de Nancy. Qui était-il ? Fils d'une grande famille de France, il était entré au Séminaire et avait étudié la théologie à Saint-Sulpice de Paris. Avec quelques confrères il avait fondé les Missionnaires de France, une de ces congrégations de prêtres qui, après la chute de Napoléon, s'étaient assigné pour but de ranimer la foi par le moyen des retraites populaires. En 1823, il était promu à l'évêché de Nancy. Très lié avec Charles $\mathrm{X}$, il avait dû, après la Révolution de 1830, quitter l'administration de son diocèse. Mais il ne restait pas sans emploi. Il redevint prédicateur; et c'est en cette qualité qu'il arrivait aux ÉtatsUnis, à l'automne de 1839. Un an plus tard, il débarquait inopinément à Québec; mais c'est Montréal qui profita le plus de son passage parmi nous. Il n'entre pas dans notre propos de raconter par le menu l'apostolat de Mgr de Forbin-Janson au Canada; nous voulons seulement dire quelle en est la portée dans l'impulsion donnée à la pratique religieuse. A ce point de vue la retraite de Montréal vient au premier rang. Ce fut un événement extraordinaire, l'événement que demandaient les circonstances et qui parut à Mgr Bourget comme un trait particulier de la Providence sur l'Église du Canada. Du 13 décembre 1840 au 21 janvier 1841, deux fois le jour la vaste église de NotreDame se remplit. Mgr de Forbin-Janson qui avait de remar- 
quables dons oratoires, laisse une part de la prédication au sulpicien de Charbonnel, surnommé le Lacordaire canadien. Si l'auditoire ne se lasse pas, si pendant cinq semaines il revient chaque jour à Notre-Dame, c'est qu'il y trouve son profit. Les instructions doctrinales sur les grandes vérités de la foi sont solides et bienfaisantes; elles sont entrecoupées de grandes cérémonies spectaculaires dans le goût de l'époque, et auxquelles la foule participe avec une telle intensité qu'elle en verse des larmes de crainte, de regret ou de joie. On estime que les deux tiers de la population catholique de Montréal ont été touchés par la retraite. Et comme dans l'autre tiers, il faut compter les catholiques de langue anglaise, on peut affirmer que pratiquement ce sont tous les fidèles de langue française qui ont été remués par la grâce. A. la messe de minuit et aux messes du jour, 4 prêtres ont distribué la sainte communion à plus de 10,000 personnes.

Événement tellement important qu'il est l'objet d'un bulletin hebdomadaire imprimé et largement répandu: résumé des sermons, description des grandes cérémonies, atmosphère de la ville en prière. Ce bulletin n'a pas peu contribué à faire naître le désir des retraites en dehors de la ville. Les demandes affluent; et comme Mgr de Nancy ne suffit pas à la tâche, Mgr Bourget s'improvise prédicateur de missions populaires et il confie cette tâche à quelques-uns de ses prêtres. Partout les résultats sont consolants, au-delà de toute espérance. On a peine à croire aujourd'hui la soif spirituelle de nos ancêtres de 1840 et les sacrifices qu'ils s'imposaient pour la satisfaire. A la retraite de Sainte-Scholastique (15-25 janvier 1841), 16 paroisses environnantes sont représentées, la plus éloignée étant celle de Bytown. On distribua 7,400 communions.

Cet accroissement de la population dans les villages de la campagne créait en hiver un problème du logement. Voici comment on le résolut en un endroit que nous n'avons pu identifier. Le texte est de Mgr de Forbin-Janson: "Les vaches étaient retirées de leurs étables pour augmenter le nombre des gîtes. On les couvrait de peaux, afin de les garantir du froid. 》 Les temples ne pouvaient pas contenir la foule: «Quand tout le 
monde est entassé dans nos églises, on voit encore se presser au dehors 800 et souvent 1000 à 1200 fidèles qui assiègent la porte, qui montent sur de gros tas de neige par pelotons de 60 ou de 80 , pour atteindre la hauteur des fenêtres, et entendre de cette manière les instructions qui se font dans l'intérieur. Et tout cela par un froid qui fige presque le sang dans les veines. » "Pendant le cours de cette année, lit-on dans les Mélanges Religieux du 12 novembre 1841, plus de 60 localités ont été favorisées d'une suite d'exercices pieux et entraînants; nos villes, Montréal, Québec et Trois-Rivières et tous nos principaux villages ont été régénérés; dans le diocèse de Québec, la grande majorité de la population a participé aux bienfaits de la retraite et dans le diocèse de Montréal, on ne trouve presque pas une seule paroisse à qui cette grâce a été offerte et dont elle n'ait profité. »Il serait exagéré de dire que toutes les têtes fortes, tous les cœurs endurcis et aigris sont revenus à la pratique religieuse. Mais l'ébranlement a été général et profond. Nous assistons là à une véritable renaissance spirituelle, à un triomphe de la foi.

\section{LES RETRAITES POPULAIRES DEVIENNENT}

UNE INSTITUTION DIOCÉSAINE

Cependant, Mgr Bourget n'était pas sans se demander si tout cela ne serait pas un feu de paille. Pour maintenir l'impulsion donnée à la foi et à la pratique religieuse, il faudrait un clergé plus nombreux, il faudrait surtout dans le diocèse une communauté de prêtres qui aurait pour but la prédication des retraites populaires. De là le voyage d'Europe qu'il entreprend au printemps de 1841. A Paris, il demande des sujets au Supérieur des Missionnaires de France, et il les veut immédiatement; grâce à la libéralité de M. Saint-Germain, curé de Saint-Laurent, le problème du logement est déjà réglé. On ne peut exaucer son désir avant deux ans; sur quoi il écrit tristement dans son Journal: "Mais cet espoir pour l'avenir ne répondait pas aux besoins du diocèse... et deux ans de délai apportaient un grand désavantage aux progrès de la réforme des mœurs, qui seule pouvait paralyser les entreprises des ministres de l'erreur. » Et 
c'est alors, quand tout espoir paraît perdu qu'il apprend à Marseille l'existence des Oblats de Marie-Immaculée, qu'il rencontre leur fondateur, Mgr de Mazenod, et qu'il voit se réaliser l'objet de son rêve. Les premiers Oblats arrivent à Montréal le 2 décembre 1841; et comme la prédication des missions populaires est leur apostolat principal, celui-ci est élevé par mandement de Mgr Bourget à la condition d'institution diocésaine. Les Oblats sont officiellement mandatés pour maintenir - et ils le font avec succès - l'œuvre si opportunément et si heureusement commencée chez nous par Mgr de Forbin-Janson. Les demandes de retraites sont adressées à l'évêché. Le nombre restreint des prédicateurs ne permettant pas d'en donner plusieurs à la fois, l'évêque assigne lui-même les dates. Et il se fait un devoir d'assister à la clôture de chaque retraite.

\section{Les Mélanges ReligieuX}

Parmi les moyens qui ont exercé une heureuse influence pendant les premières années de l'administration de Mgr Bourget, il y a lieu de signaler les Mélanges Religieux. Il serait injuste de juger cette publication d'après les normes de 1962. Dans leur courte existence (1841-1852), les Mélanges ont fait œuvre fort utile. Ils répondaient à un besoin général, car il n'existait pas à Montréal de journal franchement catholique, dévoué aux intérêts de la foi et à la défense de l'Église. Ils répondaient encore à un besoin particulier, né de la propagande protestante qui, pour entamer la masse, s'exerçait désormais en français tant à la ville qu'à la campagne. Pour les premières années au moins, on sent la rédaction préoccupée par le souci de lutter contre un ennemi réputé dangereux. Nombreux sont les faits divers relatifs aux colporteurs de bibles, nombreuses les communications où le catholicisme est vengé, la prédication des ministres suisses victorieusement attaquée. Un choix de nouvelles intelligent, actes du Souverain Pontife, conversions éclatantes, triomphes de l'Église dans les pays hérétiques, dévouement des missionnaires auprès des infidèles, allait réconforter le lecteur dans sa foi. Dans les pages qu'elle se réserve, la direction ne fait pas directement de controverse. Mais, en instruisant, elle fournit 
aux catholiques les armes dont ils ont particulièrement besoin: commentaires sur la Sainte Écriture, bulletin théologique, bulletin philosophique.

Pour le diocèse de Montréal de 1840, se lancer dans le journalisme, c'était une aventure. Elle s'explique chez Mgr Bourget par le devoir qu'il s'était imposé de réaliser les grands projets apostoliques de Mgr Lartigue. Or, celui-ci rêvait d'un journal d'information religieuse depuis 1828. Elle s'explique chez le clergé par le souci de prémunir les fidèles contre les dangers accrus de la propagande protestante.

Une double circonstance contribua aux heureux débuts de l'aventure. Quand les Mélanges commencent à paraître, janvier 1841, le grand journaliste de l'époque, Ludger Duvernay, est en exil à Burlington et La Minerve est suspendue depuis plus de trois ans. Il y avait donc place pour un hebdomadaire nouveau, d'autant plus que les autres journaux de langue française avaient peu de prestige. La seconde circonstance, c'est que la feuille diocésaine s'était fait à elle-même une belle réclame, en publiant sous le titre de Prémices des Mélanges Religieux le compte rendu de la grande retraite de Notre-Dame de Montréal.

\section{Le PREMIER Voyage DE MGR Bourget EN Europe}

$\mathrm{Du}$ succès qui avait marqué la prédication populaire de Mgr de Forbin-Janson et de ses propres visites aux quatre coins du diocèse, Mgr Bourget avait conclu que les fidèles étaient restés bons, profondément attachés à leur foi, qu'il serait facile d'en faire un peuple pratiquant, si le clergé était plus nombreux ainsi que les institutions de charité et d'éducation. Il n'était pas en son pouvoir de susciter du sol et immédiatement les uns et les autres. Mais il crut qu'il était de son devoir d'aller en chercher là où il y en avait. C'est pourquoi un an après avoir pris l'administration du diocèse, il se rendait en Europe. Nous avons dit que la venue des Oblats était un des heureux résultats de ce voyage. A la fin de cette même année, les Dames du SacréCour arrivent; le printemps suivant, ce sont les Jésuites, et en 1844, les Religieuses du Bon-Pasteur. 
Le second voyage de Mgr Bourget en Europe (1846-1847) allait attirer dans le diocèse les Clercs de Saint-Viateur, les Pères, Frères et Sœurs de Sainte-Croix.

\section{Fondations CANADIENNES}

C'était beaucoup assurément; mais ce n'était pas assez pour répondre aux besoins du diocèse en pleine expansion. En 1841, l'évêque avait espéré obtenir de France deux communautés religieuses: les Sœurs de Saint-Vincent de Paul et les Sœurs des Saints Noms de Jésus et de Marie de Marseille. Il fut amèrement déçu. Mais il ne se regarda pas comme déchargé de son devoir envers les membres souffrants de Jésus-Christ et envers une jeunesse qui montait drue. Avec les éléments qu'il avait sur place il fonda deux instituts qui n'ont cessé de grandir et de servir l'Église: les Sœurs de la Providence (1843) et les Sœurs des Saints Noms de Jésus et de Marie (1844). Enfin, il enrichissait son diocèse de deux autres fondations canadiennes: une de charité, les Sœurs de Miséricorde (1848), l'autre d'éducation, les Sœurs de Sainte-Anne (1850).

Or, qui ne le voit ? en multipliant les prêtres, en introduisant ou en fondant dans le diocèse des instituts voués aux œuvres de charité et d'éducation, Mgr Bourget donnait à la pratique religieuse une impulsion qui devait être universelle et durable.

\section{LA QUALITÉ DE NOTRE PRATIQUE RELIGIEUSE}

Cette pratique religieuse n'est pas inférieure à celle des autres pays à la même époque. Elle est entretenue par l'œuvre des retraites populaires selon la formule de la France, où la prédication des grandes vérités constitue le fond essentiel. Elle se nourrit également par la lecture des bons livres; importation ou éditions canadiennes des ouvrages qui ont connu le succès ailleurs; contre la propagande hérétique, traduction en français des lettres du grand O'Connell aux protestants wesléyens, etc. Il serait intéressant de reconstituer le catalogue d'un libraire, éditeur et imprimeur tel que Fabre. On constaterait que les livres de piété et de doctrine constituent une part considérable 
de son commerce. Cette pratique religieuse ne se borne pas à la réception annuelle des sacrements et à la messe dominicale; elle va beaucoup plus loin. La prière en famille, la récitation du chapelet, la dévotion au chemin de la croix sont en honneur.

Le culte marial est un élément essentiel de la pastorale de Mgr Bourget. En 1841, nous l'avons vu, il institue dans toutes les paroisses du diocèse l'Archiconfrérie du Très Saint et Immaculé Cœur de Marie. Au mois de juin de la même année, il visite le célèbre sanctuaire de Chartres; et quelle grâce particulière demande-t-il à la Vierge ? Celle de pouvoir restaurer la dévotion des pèlerinages à Notre-Dame de Bonsecours. En 1848, son désir est exaucé. Le mois de Marie est désormais célébré avec éclat à Bonsecours; et chaque paroisse du diocèse est invitée à y venir en pèlerinage. La définition du dogme de l'Immaculée Conception fut l'occasion d'une plus grande impulsion donnée dans le diocèse à la piété mariale.

L'heure n'était pas encore à la communion fréquente et quotidienne. Les cahiers de prônes nous apprennent que les fidèles étaient invités à s'approcher de la Sainte table environ 10 fois par année, c'est-à-dire aux grandes fêtes liturgiques, à la fête du patron de la paroisse, le jour de la première communion des enfants; à quoi il faudrait ajouter les communions prescrites par les règles particulières des confréries ou des associations pieuses. Mais si les communions sont plus rares qu'aujourd'hui, le culte du Très Saint-Sacrement est entouré du plus profond respect. En 1850, l'évêque institue l'Adoration perpétuelle qui assure au Saint-Sacrement des adorateurs perpétuels, et dont les membres organisent les processions de la Fête-Dieu, et, tour à tour, accompagnent le prêtre, quand il va porter le bon Dieu aux malades. Mgr Bourget a le mérite d'avoir introduit au Canada la dévotion des Quarante-Heures (1857). Cette exposition solennelle du Saint-Sacrement était un événement paroissial, l'occasion d'une prédication doctrinale plus soignée, de communions plus ferventes et plus nombreuses. Si les décrets de saint Pie $\mathrm{X}$ sur la communion fréquente n'ont pas rencontré chez nous la résistance qu'on leur a opposée en certains pays de vieille chrétienté, c'est que nous étions prêts à les recevoir. 
La Société et le Séminaire des Missions-Étrangères, l'apostolat actuel des nôtres dans les cinq continents du monde, et qui est une des plus belles pages de notre histoire, sont des résultats, pour une large part, de l'esprit missionnaire que Mgr Bourget a inlassablement prêché de parole et d'exemple. La générosité des fidèles pour leurs œuvres de charité atteste également la qualité de leur pratique religieuse. Enfin, les parents regardent comme un honneur les vocations sacerdotales ou religieuses de leurs enfants. Celles-ci sont nombreuses, et permettent à Montréal non seulement de répondre à ses propres besoins, mais de se placer au premier rang des diocèses missionnaires de l'Amérique du Nord.

Nous n'avons pas à rougir de la pratique religieuse de nos ancêtres. Leur piété et leur foi étaient de leur époque; moins spectaculaires que les nôtres, à certains égards, elles ne manquaient ni de sérieux ni de profondeur. Cette pratique religieuse a produit des œuvres et des institutions qui font encore la gloire de l'Église; des hommes d'Église et des laïcs militants d'une qualité nettement supérieure à la moyenne de l'époque.

\section{LA PART DES LAÏQUES}

On peut dire qu'à partir de 1860 , les dangers provenant de la propagande protestante ou de l'indifférence religieuse ont pratiquement disparu. A la ville comme à la campagne, il y a encore des récalcitrants; mais ils sont le petit nombre, l'exception. Et cela n'empêche pas de dire que, dans son ensemble, le diocèse de Montréal est catholique et catholique pratiquant. Un tel progrès en un temps relativement court, en un temps aussi où le diocèse de Montréal connaît une heureuse crise de croissance et commence une évolution qui en fera un diocèse urbain, commercial et industriel, est tout à l'honneur de Mgr Bourget et de ses collaborateurs immédiats. Mais il est juste de remarquer qu'une large part du mérite revient aux laïques. Dès le début, Mgr Bourget sut intéresser, associer tous les fidèles à la vie et au progrès de leur Église. Au moment où il décide de faire son premier voyage en Europe, il est littéralement sans le sou. Dans la Pastorale où il annonce son départ, il fait appel à la charité 
de tout le diocèse, parce que, dit-il, c'est tout le diocèse qui est intéressé au succès. Même disette d'argent quand il est question de construire l'Asile de la Providence pour les femmes âgées et infirmes. Comme l'institution est destinée au bien de toute la ville, c'est à la charité de la ville d'en faire les frais. Accompagné de deux laïques, il va lui-même solliciter l'aumône de porte en porte. Il recueille ainsi la somme, considérable pour l'époque, de $\$ 4,000$. Mais surtout, il a prêché de parole et d'exemple la belle vertu de charité. Sans le secours de laïques riches et influents, sa bonne volonté aurait été souvent paralysée. Citons un nom: Olivier Berthelet, dont on a dit qu'il avait donné aux œuvres et aux institutions de l'évêque une somme équivalant à $\$ 1,000,000$. d'aujourd'hui.

Les Sœurs de la Providence sont nées d'une institution charitable laïque: les Dames de la Charité. Or, les Dames de la Charité ne cessent pas d'exister après la fondation de l'Institut; elles restent intimement unies à celui-ci. Dans son estime et son affection, Mgr Bourget ne les sépare jamais des religieuses. Les Sœurs de la Providence étaient, à l'origine, comme un élément de solution de la question sociale. Chaque maison avait ses sœurs quêteuses qui, le panier au bras, allaient chercher chez les riches des dons en argent et en nature qu'elles distribuaient ensuite aux pauvres.

En 1842, l'évêque instituait par mandement dans toutes les paroisses du diocèse des Sociétés de Tempérance et de Charité, dont le curé était l'aumônier, mais dont l'administration était laïque. Les Sociétés de Charité avaient un double but: d'abord subvenir aux besoins des pauvres de la paroisse; car Mgr Bourget voulait diminuer, enrayer même la plaie sociale et morale des mendiants itinérants. Elles avaient pour seconde tâche de placer dans des familles honnêtes les jeunes de la campagne que la nécessité amenait en ville. Si l'on ajoute à cela que l'Association de la Propagation de la Foi et les Sociétés de Saint-Vincent de Paul avaient des conseils de direction et d'administration laïques actifs et efficaces, on admettra que le laïcat a tenu un rôle important, parfois irremplaçable, sous l'administration de Mgr Bourget. 


\section{CONCLUSION}

Nous n'avons parlé jusqu'ici que de l'impulsion donnée par Mgr Bourget à la pratique et à la vie religieuse dans le diocèse de Montréal. Mais il ne faudrait pas oublier que les diocèses actuels d'Ottawa, des Trois-Rivières, de Saint-Hyacinthe, de Sherbrooke, de Valleyfield, de Pembroke, de Joliette, de MontLaurier, de Saint-Jean-de-Québec et de Saint-Jérôme étaient contenus en tout ou en partie dans les limites du diocèse de Montréal de 1840. Le diocèse de Québec était solidement organisé; plus homogène que celui de Montréal, il était moins exposé que celui-ci à la propagande protestante et les Troubles de 1837 y avaient moins aigri les esprits. Il a cependant, lui aussi, profité du renouveau spirituel de Montréal. C'est par suite de l'exemple de Mgr Bourget, avec son encouragement et sa bénédiction que les Oblats, les Frères des Écoles Chrétiennes, les Jésuites, les Sœurs Grises se sont établis à Québec.

Le reste du pays, depuis les Provinces Maritimes jusqu'aux Montagnes Rocheuses, la Nouvelle-Angleterre, les Etats américains de la Côte du Pacifique ont également recueilli, et avec abondance, les fruits de cette impulsion donnée à la pratique religieuse qui eut pour centre Montréal et pour âme dirigeante Mgr Ignace Bourget. 